\title{
Four point bending setup for characterization of semiconductor piezoresistance
}

\author{
Richter, Jacob; Arnoldus, Morten Berg; Hansen, Ole; Thomsen, Erik Vilain
}

Published in:

Review of Scientific Instruments

Link to article, DOI:

$10.1063 / 1.2908428$

Publication date:

2008

Document Version

Publisher's PDF, also known as Version of record

Link back to DTU Orbit

Citation (APA):

Richter, J., Arnoldus, M. B., Hansen, O., \& Thomsen, E. V. (2008). Four point bending setup for characterization of semiconductor piezoresistance. Review of Scientific Instruments, 79(4), 044703.

https://doi.org/10.1063/1.2908428

\section{General rights}

Copyright and moral rights for the publications made accessible in the public portal are retained by the authors and/or other copyright owners and it is a condition of accessing publications that users recognise and abide by the legal requirements associated with these rights.

- Users may download and print one copy of any publication from the public portal for the purpose of private study or research.

- You may not further distribute the material or use it for any profit-making activity or commercial gain

- You may freely distribute the URL identifying the publication in the public portal

If you believe that this document breaches copyright please contact us providing details, and we will remove access to the work immediately and investigate your claim 


\title{
Four point bending setup for characterization of semiconductor piezoresistance
}

\author{
J. Richter, ${ }^{\text {a) }}$ M. B. Arnoldus, O. Hansen, ${ }^{\text {b) }}$ and E. V. Thomsen \\ Department of Micro- and Nanotechnology, Technical University of Denmark, \\ DTU Nanotech Building 345 East, DK-2800 Kongens Lyngby, Denmark
}

(Received 30 November 2007; accepted 24 March 2008; published online 16 April 2008)

\begin{abstract}
We present a four point bending setup suitable for high precision characterization of piezoresistance in semiconductors. The compact setup has a total size of $635 \mathrm{~cm}^{3}$. Thermal stability is ensured by an aluminum housing wherein the actual four point bending fixture is located. The four point bending fixture is manufactured in polyetheretherketon and a dedicated silicon chip with embedded piezoresistors fits in the fixture. The fixture is actuated by a microstepper actuator and a high sensitivity force sensor measures the applied force on the fixture and chip. The setup includes heaters embedded in the housing and controlled by a thermocouple feedback loop to ensure characterization at different temperature settings. We present three-dimensional finite element modeling simulations of the fixture and discuss the possible contributions to the uncertainty of the piezoresistance characterization. As a proof of concept, we show measurements of the piezocoefficient $\pi_{44}$ in $p$-type silicon at three different doping concentrations in the temperature range from $T=30{ }^{\circ} \mathrm{C}$ to $T=80{ }^{\circ} \mathrm{C}$. The extracted piezocoefficients are determined with an uncertainty of 1.8\%. (C) 2008 American Institute of Physics. [DOI: 10.1063/1.2908428]
\end{abstract}

\section{INTRODUCTION}

Since the pioneering work of Smith in $1954,{ }^{1}$ piezoresistivity of silicon has attracted attention from both academia ${ }^{2-4}$ and industry. ${ }^{5,6}$ Smith experimentally determined the three piezoresistance coefficients of lightly doped silicon. The piezoresistance coefficients for more heavily doped silicon were later experimentally determined by other research groups, ${ }^{2,3}$ and still, today, the piezoresistance coefficients of silicon and other materials are topics of interest in both academia $^{4,7,8}$ and industry. The continued academic interest is partly due to the scarcity of reliable measurements and partly due to a discrepancy between theoretical models and available measurements especially for $p$-type silicon. ${ }^{9-12}$

Essentially, the piezoresistance effect is a change in the resistivity tensor (second order) caused by an applied stress. ${ }^{13}$ The effect is characterized by a fourth order piezoresistivity tensor, which, in the case of silicon due to symmetry, has three independent coefficients. ${ }^{14}$ The piezoresistance coefficients are dependent on sample temperature, doping level, and doping type. ${ }^{2}$ In order to measure these coefficients and characterize the effect, it is necessary to apply a well controlled stress to the silicon sample with well defined resistors and measure the relative change in resistance of these.

In the original experiments by Smith, ${ }^{1}$ silicon rods were pulled to apply a uniform uniaxial stress. Machined pull samples with through holes were used in Ref. 2 and a pull force was applied by pins inserted in the through holes.

For microfabricated thin film devices, it is more conve-

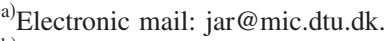

b) Also at CINF-Center for Individual Nanoparticle Functionality, Department of Micro- and Nanotechnology, Technical University of Denmark, DTU Nanotech Building 345 East, DK-2800 Kongens Lyngby, Denmark.
}

nient to use a four point bending (4PB) fixture. ${ }^{8,15-19}$ In Refs. 16 and 18 an optical method is used to measure the deflection and curvature of the chip. The stress is applied to the chip using a piezoelectric actuator and a translation stage, respectively. In Ref. 17, the displacement of the chip is known at the contact points between chip and $4 \mathrm{~PB}$ fixture and this enables a calculation of the applied stress in the chip. Reference 8 and 15 use simple loads to apply the force and has no external measurement of the applied force. This is a cumbersome and time consuming method, especially for characterization at different temperatures.

We present a four point bending method where a motorized stepper actuator is used to apply a displacement while the force on the chip sample is measured with a dedicated force sensor. With this method, the measured force can be directly applied to calculate the stress. Thus, Young's modulus is not included in the stress calculation as is the case when a deflection is measured. The compact setup has a total volume of $635 \mathrm{~cm}^{2}$.

The four point bending setup is designed and fabricated to analyze the piezoresistance coefficients of embedded piezoresistors located on a dedicated silicon chip. The main focus is to characterize the piezoresistivity of $p$-type silicon and other related semiconductor materials, e.g., Si under tensile strain ${ }^{19}$ and compressively strained SiGe. ${ }^{8}$ In this paper, we present measurements of the piezocoefficient $\pi_{44}$ in $p$-type silicon with several different doping concentrations in the temperature range $T=30-80{ }^{\circ} \mathrm{C}$ as an example of use of the setup. Boron doped silicon is the preferred piezoresistive material in commercial micro electromechanical systems (MEMS) due to the large piezocoefficient $\pi_{44}$ and the very low values of the two other piezocoefficients $\pi_{11}$ and $\pi_{12}$. When the piezoresistors are directed along certain crystal directions, $\langle 110\rangle$, and placed in a Wheatstone bridge configu- 


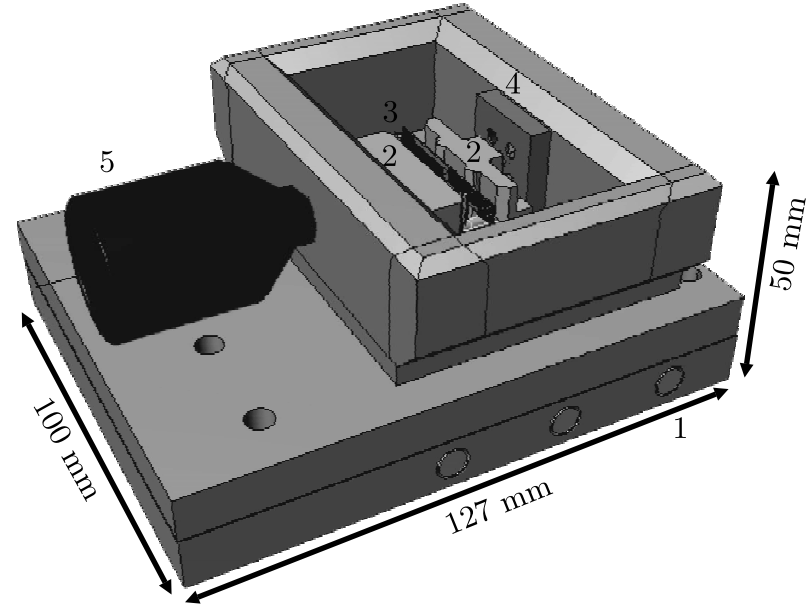

FIG. 1. Schematic of the complete piezoresistance characterization setup. The setup comprises cartridge heaters (1) that are embedded in the bottom plate of the Al housing surrounding the 4PB fixture (2) consisting of base and slider, the chip (3), and the force sensor (4). The actuator motor (5) is placed outside the Al housing to prevent heating of the motor.

ration, these piezocoefficients result in a high sensitivity of the MEMS device, since the effective longitudinal and transverse piezocoefficients are large and almost matched in magnitude but of opposite sign.

We characterize the $4 \mathrm{~PB}$ setup using analytical expressions, finite element modeling (FEM), calibration measurements, and an application specific stress sensor chip. The stress distribution in a chip in the 4PB setup is thoroughly investigated and this analysis is used to estimate the uncertainties of the measured piezocoefficients.

\section{APPARATUS}

The piezoresistance characterization setup consists of a 4PB fixture with integrated thermocouples and temperature control. An actuator applies a displacement to the fixture and the force is measured by a force sensor. A schematic of the setup is shown in Fig. 1.

The fixture is placed in an aluminum housing including a metal lid (not shown in the figure) to stabilize temperature and shield off light. The thickness of the aluminum bottom plate and sidewalls are 10 and $20 \mathrm{~mm}$, respectively. Aluminum has a very high thermal conductivity of $239 \mathrm{~W} / \mathrm{m} \mathrm{K}^{-1}$ (Ref. 20) compared to air $\left(\approx 0.02 \mathrm{~W} / \mathrm{m} \mathrm{K}^{-1}\right)$, ensuring a uniform heat distribution in the aluminum casing.

The 4PB fixture is guided along the axis of the actuator by small rails in the bottom plate of the setup. The contact area between the rails and the $4 \mathrm{~PB}$ fixture is very small $\left(40 \mathrm{~mm}^{2}\right)$ which minimizes friction between fixture and bottom plate. The frictional force may be even lower in a vertical design, however, the horizontal design was chosen in order to use gravity to assist alignment of the different parts of the setup and of the sample.

\section{A. Four point bending fixture}

An exploded view of the 4PB setup is shown in Fig. 2. It consists of a base part containing two blades separated by a distance of $28 \mathrm{~mm}$ and a sliding part where two more blades are formed separated by a distance of $12 \mathrm{~mm}$. The base part

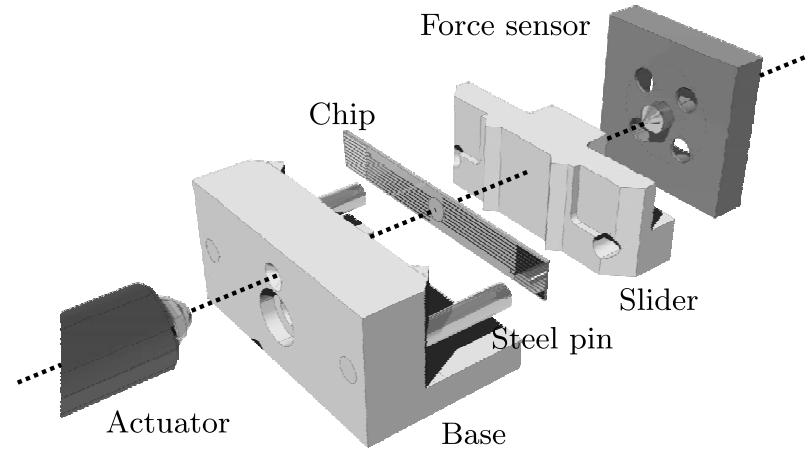

FIG. 2. Exploded view of the setup illustrating the uniaxial force interaction between actuator, 4PB fixture, chip, and force sensor. This ensures uniformity of the stress in the center region of the chip.

slides on two rails formed in the bottom plate of the setup casing. The sliding part is in contact with the base only at two steel pins that ensure good directional control of the force and low frictional force. A dedicated silicon chip is placed in between the sliding part and the base part; the force is applied to the chip by the four blades.

The 4PB fixture applies a uniaxial and uniform stress distribution to the center region of the chip. The bending force $F$ is measured using a force sensor. From simple bending beam theory, we obtain the stress $\sigma_{x x}$ (Ref. 8)

$$
\sigma_{x x}=\frac{6 F a z}{w h^{3}},
$$

where $h$ is the thickness of the chip, $z$ is the position of the resistor with respect to the neutral plane of the chip, i.e., $z=h / 2$ at the surface, $a$ is the distance between the inner and outer blades of the 4PB fixture, and $w$ is the width of the chip.

The 4PB fixture is made from the thermoplastic material polyetheretherketon (PEEK) which is a semicrystalline material. Young's modulus of PEEK is $Y=3.5 \mathrm{GPa}$ and the mechanical properties are quite stable in the temperature range from -64 to $250{ }^{\circ} \mathrm{C}$. ${ }^{21}$

The vertical alignment of the chip to the $4 \mathrm{~PB}$ fixture is ensured by resting the chip on the two steel pins in the setup (see Fig. 2). Horizontal alignment of the chip is done by visual inspection. The stress is considered constant in the area between the two inner blades on the slider. The dimensions of the resistor are much smaller than the $12 \mathrm{~mm}$ distance between the two inner blades. Thus, the resistors which are located in the middle region between the two inner blades experience a uniform stress.

\section{B. Force sensor}

The force sensor is a Strain Measurement Devices s415 button cell. ${ }^{22}$ It consists of a plate with four sputter defined resistors in a Wheatstone bridge configuration. The force sensor is fastened to the setup casing. An input voltage, $V_{\text {in }}$ $=10 \mathrm{~V}$, is applied to the bridge. The output voltage of the Wheatstone bridge, $V_{o}$, depends linearly on the force $F$, as described by 


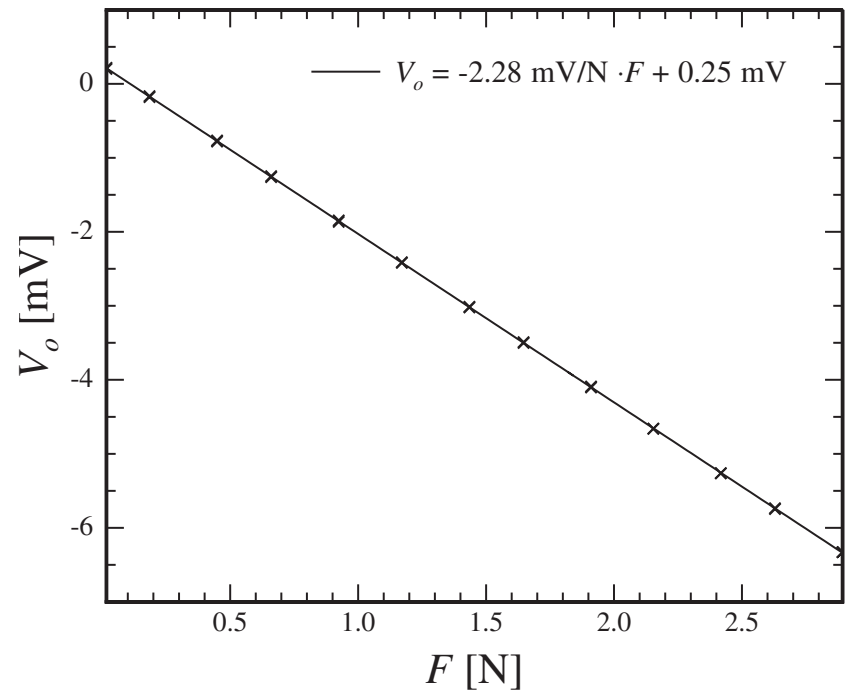

FIG. 3. Force sensor calibration curve at $T=30^{\circ} \mathrm{C}$. A force $F$ is applied by adding weights to the force sensor with an input voltage $V_{\text {in }}=10 \mathrm{~V}$ while the output voltage, $V_{o}$ of the Wheatstone bridge is measured. The slope and offset of linear fit (solid line) at different temperatures are listed in Table I.

$$
F=\frac{1}{\alpha}\left(V_{o}-V_{\text {off }}\right),
$$

where $1 / \alpha$ is the constant of proportionality and $V_{\text {off }}$ is the offset voltage.

The constant $\alpha$ is measured in a calibration setup where the force sensor is horizontally placed and well known forces are applied using weights of different masses. The calibration curve at $T=30{ }^{\circ} \mathrm{C}$ is shown in Fig. 3, where the calibration constant, $\alpha$, is determined as the slope of the linear fit. The force sensor showed a small hysteresis in the output when increasing and decreasing the applied force. The hysteresis is described by a $0.3 \%$ change in the calibration constant and this will not significantly contribute to the uncertainty. The force sensor was calibrated at different temperatures (see Table I). The temperature dependence of $\alpha$ is significant and the values in Table I are included in the analysis.

The force sensor offset is a result of imbalance in the thin film Wheatstone bridge and the actual force sensor temperature. This offset has no influence on the measurements since the force sensor is offset compensated before the actual measurement.

\section{Actuator}

The motorized Newport NSA12 microstep actuator ${ }^{23}$ used in the setup has a resolution of $0.3 \mu \mathrm{m}$ and a maximum loading capacity of $25 \mathrm{~N}$. The actual force on the actuator is

TABLE I. Force sensor calibration. The table summarizes the slope $\alpha$ and offset $V_{\text {off }}$ of the linear fits to experimentally obtained force-voltage curves at four different temperatures with an input voltage of $V_{\text {in }}=10 \mathrm{~V}$.

\begin{tabular}{ccc}
\hline \hline$T\left({ }^{\circ} \mathrm{C}\right)$ & $V_{\text {off }}(\mathrm{m} \mathrm{V})$ & $\alpha(\mathrm{m} \mathrm{V} / \mathrm{N})$ \\
\hline 30 & 0.25 & 2.280 \\
47 & 0.10 & 2.290 \\
64 & -0.05 & 2.294 \\
81 & -0.20 & 2.312 \\
\hline \hline
\end{tabular}

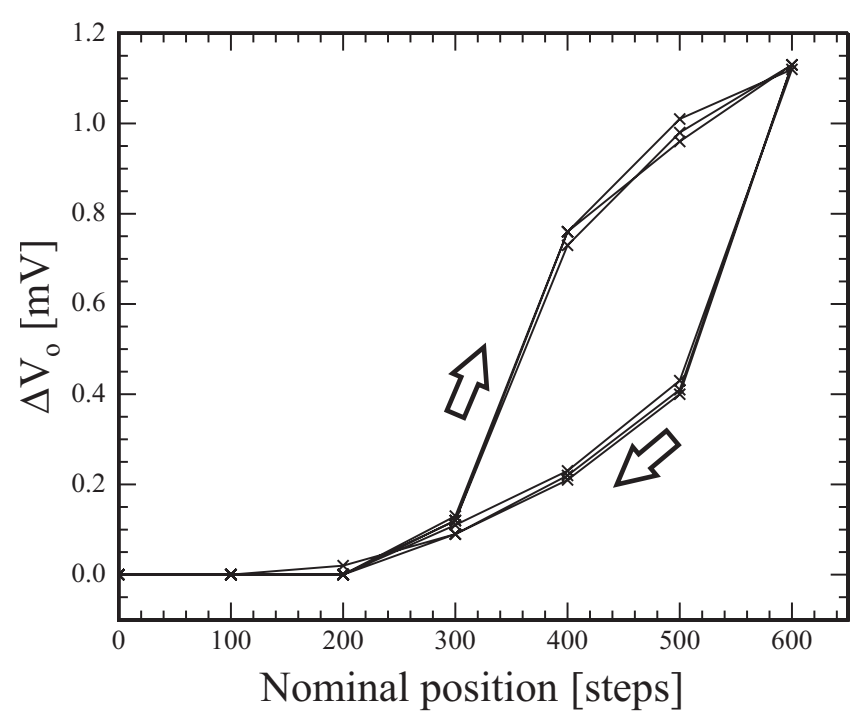

FIG. 4. Actuator position hysteresis. The force sensor output voltage $\Delta V_{o}=V_{o}-V_{\text {off }}$ as a function of the nominal actuator displacement in three subsequent measurement series. The output voltage depends on the direction of the actuator motion due to a hysteresis in the actual actuator position.

of no interest since the force on the chip is measured by an independent force sensor. Characterization of the actuator has shown severe hysteresis during increasing and subsequent decreasing loads. The displacement of the actuator is affected by the force thus the actual position of the actuator is not reliable (see Fig. 4). The actuator is, in turn, increasing the load on the chip and decreasing the load while the output voltage on the force sensor is measured. Since previous characterization in Fig. 3 showed that the force sensor signal linearly depends on the applied force, we conclude that the hysteresis is caused by the actuator. This actuator hysteresis has no influence on the piezoresistance measurements since the force sensor signal (and not the actuator displacement) is used in the characterization.

\section{Temperature monitor and control}

The setup contains two integrated thermocouples. One thermocouple is placed in air close to the chip and reads the temperature near the chip. The other thermocouple is attached to the aluminum casing. This thermocouple supplies a feedback signal to the temperature controller. The thermocouples have small thermal masses resulting in a fast response time. The temperature is read using a Pico Technologies data logger. ${ }^{24}$

A Watlow series 96 temperature controller is used to control the temperature and a Watlow solid state DIN-AMITE power relay supplies bias current to three Watlow cartridge heaters embedded in the aluminum plate placed below the setup, as seen in Fig. 1. The actuator has a temperature operating range between 5 and $40{ }^{\circ} \mathrm{C}$, thus cooling of the actuator is necessary. This is done using an ARX Cera Dyne fan and a heat sink on the actuator. A metal shield between the fan and the metal casing prevents significant cooling of the setup casing.

The time to reach a given temperature is measured to be less than $t_{\text {eq }}=20 \mathrm{~min}$. This is done by measuring the temperature inside the $\mathrm{Al}$ housing as a function of time at a 


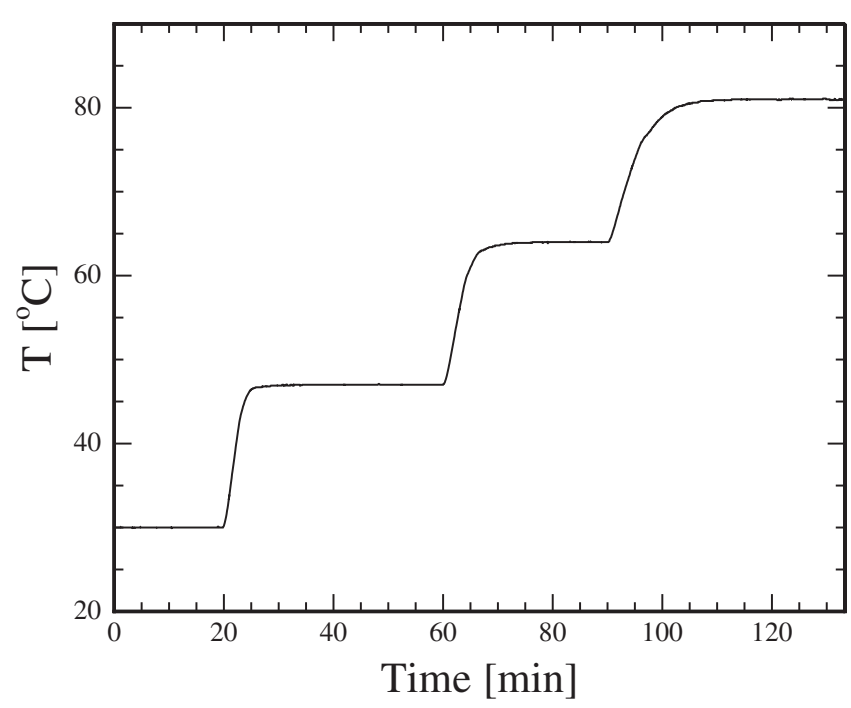

FIG. 5. The temperature readout from the thermocouple placed inside the aluminum housing near the chip as a function of time. The temperature is sequentially set to the values $T=30,47,64$, and $81{ }^{\circ} \mathrm{C}$. The maximum time for equilibrium is $t_{\mathrm{eq}}=20 \mathrm{~min}$ seen when the temperature is increased from 64 to reach $81{ }^{\circ} \mathrm{C}$.

given set temperature. Figure 5 shows the temperature development at set temperatures $30,47,64$, and $81^{\circ} \mathrm{C}$. The maximum equilibration time is $t_{\mathrm{eq}}=20 \mathrm{~min}$ when increasing the temperature by $17{ }^{\circ} \mathrm{C}$ from $64{ }^{\circ} \mathrm{C}$. All measurement series are performed by increasing the temperature in steps of $10^{\circ} \mathrm{C}$, thus thermal equilibrium is reached within less than $20 \mathrm{~min}$.

\section{E. Chip design}

The silicon chip to be inserted in the setup is a $4 \mathrm{~cm}$ long and $5.3 \mathrm{~mm}$ wide beam. The resistors are fabricated on $350 \mu \mathrm{m}$ (001) silicon on insulator wafers with a device layer of $2 \mu \mathrm{m}$. The device layer is thinned down to $500 \mathrm{~nm}$ by oxidation thinning. The piezoresistors are formed in the $500 \mathrm{~nm}$ thick device layer by boron doping using ion implantation. The implanted doses were $D=1.5 \times 10^{13}, 1.5$ $\times 10^{14}$, and $1.5 \times 10^{15} \mathrm{~cm}^{-2}$, respectively, all at an energy of $50 \mathrm{keV}$. The resulting final doping concentrations in the samples are then $N_{A}=1.5 \times 10^{17}, 2.0 \times 10^{18}$, and 2.2 $\times 10^{19} \mathrm{~cm}^{-3}$, respectively. The fabrication of the piezoresistors was performed by using a long postoxidation annealing which activates the acceptors and results in an extremely uniform doping profile in the piezoresistors, as verified in a simulation using the SILVACO ATHENA process simulator. ${ }^{25}$ The piezoresistors are patterned using UV lithography and reactive ion etching (RIE). Contact windows in the oxide are formed using buffered HF on a photoresist mask. This mask is also used to pattern an additional high dose $\left(5 \times 10^{15} \mathrm{~cm}^{-2}\right)$ boron ion implant to improve the contact resistance. A Ti/ Al metal layer is deposited in a lift-off process to form interconnects and electrical contact to the piezoresistors. Finally, the chips are diced in a deep RIE using the Bosch process ${ }^{26}$ with an etch angle of $90^{\circ} \pm 1^{\circ}$ to accurately define the chip direction with respect to the crystal orientation. A cross sectional schematic of the chip is shown in Fig. 6.

The chip layout is sketched in Fig. 7. This test chip is

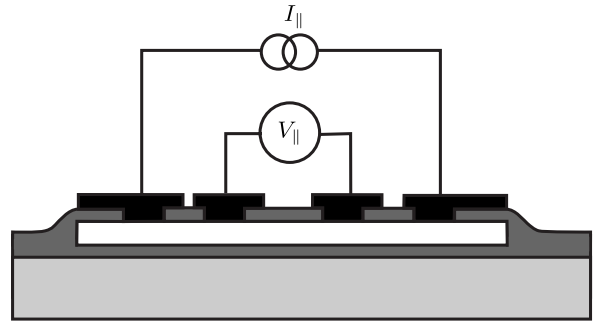

FIG. 6. Schematic cross section of the chip. The silicon piezoresistor (white) is surrounded by silicon dioxide (dark gray) and electrically connected by $\mathrm{Al} / \mathrm{Ti}$ metal tracks (black) on a silicon substrate (light gray). The electrical resistance measurement is performed by a four terminal high impedance voltage measurement where a current $I_{\|}$is forced through the resistor from the outer contacts while a voltage drop $V_{\|}$is measured on the inner contacts.

designed to measure the piezocoefficient $\pi_{44}$ and the sum of the two other coefficients $\pi_{11}+\pi_{12}$ in $p$-type silicon. The relative resistance change, $\Delta R / R$, in a resistor with an applied uniaxial stress, $\sigma_{x x}$, is given by ${ }^{8}$

$$
\frac{\Delta R}{R}=\sigma_{x x}\left[\frac{\pi_{11}+\pi_{12}+\pi_{44} \cos (2 \theta)}{2}\right],
$$

where $R=V_{\|} / I_{\|}$is the resistance according to Figs. 6 and 7(b) and $\theta$ is the angle of the resistor direction with respect to the $\sigma_{x x}$ stress direction, i.e., [110] according to Fig. 7(a). By plotting the relative resistance change as a function of the applied stress, we obtain a value of the bracketed piezocoefficient linear combination in Eq. (3) for each resistor. This value is plotted as a function of $\cos (2 \theta)$ to determine the piezocoefficient $\pi_{44}$ as the slope of a linear fit and the sum $\left(\pi_{11}+\pi_{12}\right)$ as the offset of a linear fit.

\section{F. Electrical measurements}

The full electrical setup is sketched in Fig. 8. A thermocouple placed in the Al housing supplies the signal to a feedback loop for the heaters in the bottom of the Al housing through the temperature controller. A Keithley 2400 sourcemeter and a Keithley 2700 multimeter are used for the electrical measurements on the chip with a simple four ter-

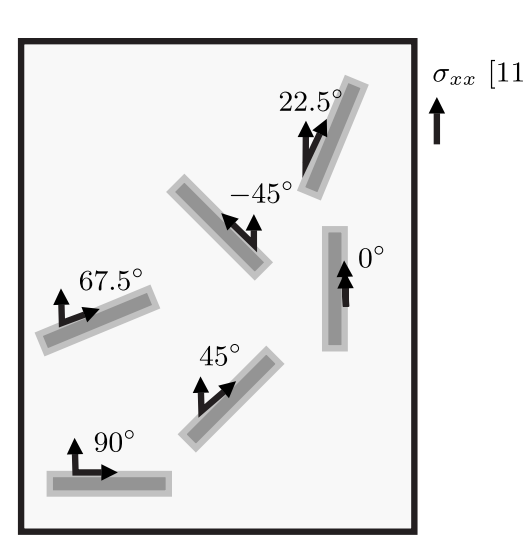

(a)

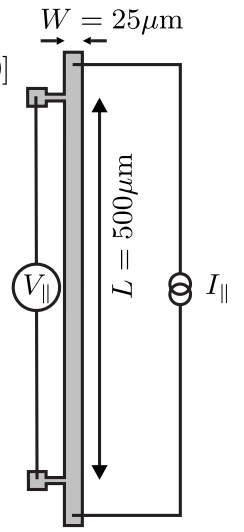

(b)
FIG. 7. Chip design for piezoresistance measurements, not to scale. (a) The chip has six resistors all placed in the center region of the chip. The resistors are oriented along the different angles with respect to the [110] stress direction. (b) A close-up of the resistor showing the four terminal electrical resistance measurement used on each resistor. 


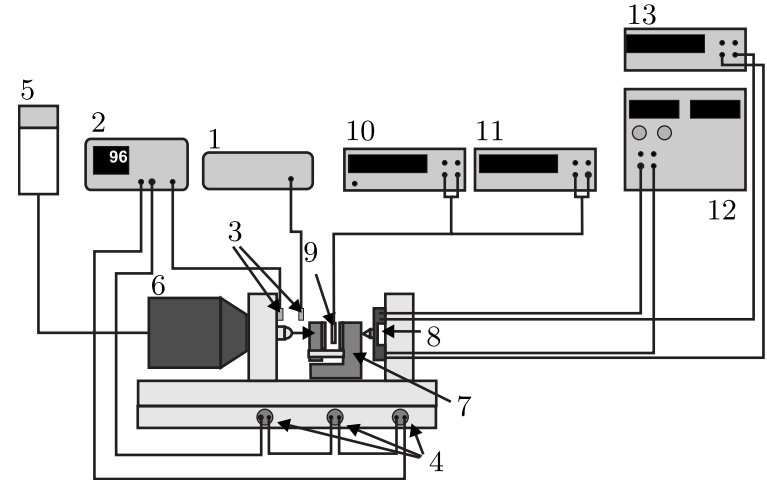

FIG. 8. A schematic of the complete setup. (1) Temperature logger. (2) proportional-integral-derivative temperature controller. (3) Thermocouples. (4) Cartridge heaters. (5) Motion controller. (6) Actuator. (7) 4PB fixture. (8) Force sensor. (9) Chip. (10) Keithley 2700 Multimeter with multiplexer. (11) Keithley 2400 sourcemeter. (12) TTi EL302T triple Power Supply. (13) Keithley 2000 multimeter.

minal measurement, as illustrated in Fig. 6. The force sensor is connected to a power supply and a multimeter. All instruments are controlled via a NATIONAL INSTRUMENTS LABVIEW software interface.

The chip is contacted using zero insertion force flat flexible cable (FFC) connectors [Molex Electronics, part No. 52746-1090 (Ref. 27)], as shown in Fig. 9. The connectors do not influence the stress distribution in the chip and allow for mechanical movement of the chip.

\section{ERROR ESTIMATES}

The accuracy of the piezoresistance measurements depends on several factors associated with the $4 \mathrm{~PB}$ fixture. First, the inaccuracy of the intended uniaxial stress caused by force and geometry errors is discussed. Second, deviations from the assumed uniaxial stress distribution caused by model insufficiencies and alignment errors are analyzed using FEM and analytical approaches. Third, we discuss the contribution from errors due to the electronic equipment. Finally, the frictional forces in the setup are outlined and their contribution to the piezoresistance measurement is discussed.

\section{A. Stress uncertainty}

The stress at the surface $(z=h / 2)$ is determined by Eq. (1), thus the relative uncertainty in the applied stress is given by

$$
\frac{\Delta \sigma_{x x}}{\sigma_{x x}}=\sqrt{\left(\frac{\Delta F}{F}\right)^{2}+\left(\frac{\Delta a}{a}\right)^{2}+2\left(\frac{\Delta h}{h}\right)^{2}+\left(\frac{\Delta w}{w}\right)^{2}} .
$$

The uncertainty of the applied force $F$ we obtain from the standard deviation on the calibration factor $\alpha$ and an estimate of the precision of the actual electrical measurement on the force sensor. This gives an uncertainty on the measured force of $0.25 \%$. The distance between the inner and outer blades in the 4PB fixture is $a=8.0 \mathrm{~mm}$ with an uncertainty of $\Delta a=0.1 \mathrm{~mm}$. The chip thickness is $h=356 \mu \mathrm{m}$ with an uncertainty of $\Delta h=2 \mu \mathrm{m}$. The chip dimensions are individually measured. The width of the chip, $w=5.3 \mathrm{~mm}$, is accurately defined by photolithography, as described in Sec. II E and the uncertainty can be assumed to be negligible compared to the

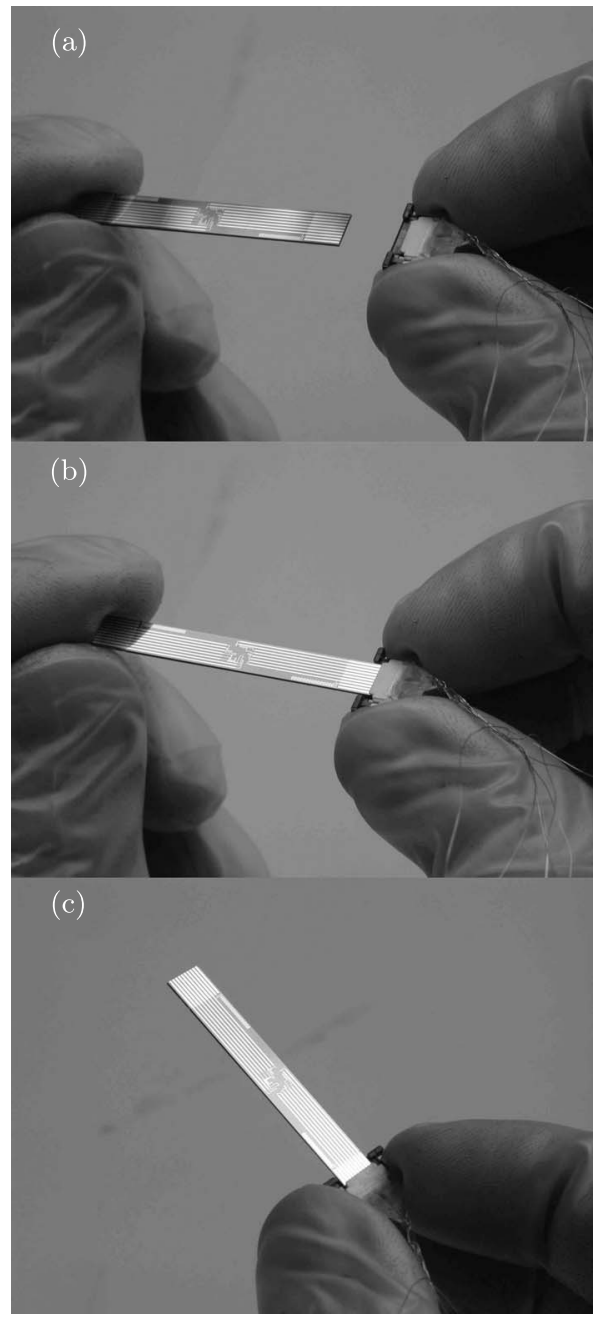

FIG. 9. The chip is electrically interfaced through zero insertion force flat flexible cable (FFC) connectors. The assembly is an easy "plug and measure" method, (a) before insertion of chip in the connector, (b) during insertion, and (c) the chip is connected to the electronic instrumentation.

above values. The vertical sidewalls of the chip are obtained from the deep RIE to have an angle of $90^{\circ} \pm 1^{\circ}$. This angle results in a negligible difference of the width of $2 \times 6 \mu \mathrm{m}^{2}$ on front side compared to back side of the chip. Thus, the relative uncertainty of the induced stress is

$$
\frac{\Delta \sigma_{x x}}{\sigma_{x x}}=\sqrt{(0.0025)^{2}+\left(\frac{0.1}{8.0}\right)^{2}+2\left(\frac{2}{356}\right)^{2}}=1.5 \% .
$$

\section{B. Stress distribution: FEM analysis}

The stress distribution in the chip is simulated in COMSOL MULTIPHYSICS 3.3. In the simulation, the inner blades of PEEK are constrained in the $z$ direction on the bottom surface of the PEEK mass. The outer blades each have a distributed force, $F / 2$ on the surface plane. The chip is assigned the elastic parameters Young's modulus $Y=170 \mathrm{GPa}$ and Poisson's ratio $\nu=0.07$ from Ref. 28 , since the chip is stressed along the [110] direction. Figure 10(a) shows the stress distribution $\sigma_{x x}$ in the chip at a force of $F=2.5 \mathrm{~N}$. The resistor area in the center region of the chip is sketched by the dashed square $\left(3 \times 3 \mathrm{~mm}^{2}\right)$. The in-plane stress distribu- 
(a)

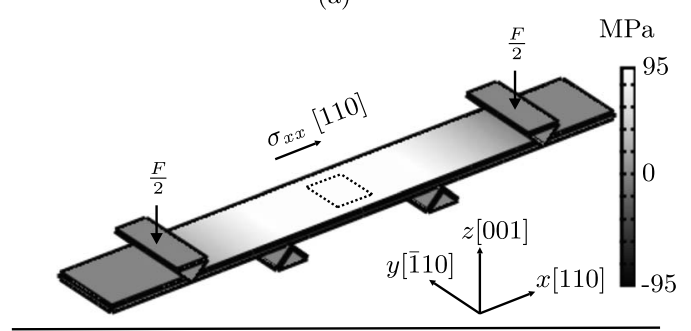

(b)

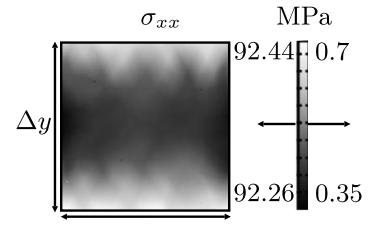

$\Delta x$

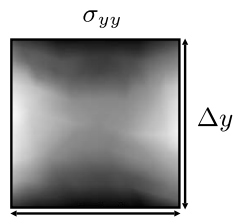

$\Delta x$

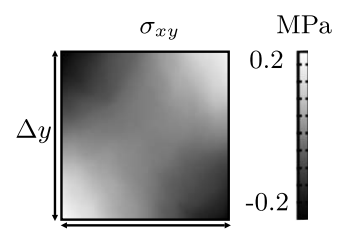

$\Delta x$

FIG. 10. FEM analysis of the 4PB setup. (a) Gray-scale encoded stress distribution $\sigma_{x x}$ in the chip placed in the 4PB fixture with an applied force $F$. The area marked by a dashed square $\left(\Delta x \times \Delta y=3 \times 3 \mathrm{~mm}^{2}\right)$ indicates the location of the piezoresistors in the surface of the chip center. In (b), a zoom-in on this area shows all in-plane stress distributions, $\sigma_{x x}, \sigma_{y y}$, and $\sigma_{x y}$. Notice the different scales, the stress $\sigma_{x x}$ is more than 100 times larger than $\sigma_{y y}$ and $\sigma_{x y}$.

tions $\left(\sigma_{x x}, \sigma_{y y}\right.$, and $\left.\sigma_{x y}\right)$ in the resistor area are shown in Fig. 10(b).

Since the chip is subjected to pure bending, we expect an exact solution to the problem with $\sigma_{x x}$ given by Eq. (1) and all other stress components to be zero for a narrow chip. However, due to the rather large width to length ratio, $w / l$ $=0.13$, the chip is also subjected to a transverse stress. As is seen in Fig. 10(b), this transverse stress is at least a factor 100 less than the stress $\sigma_{x x}$.

Ideally, the blades are considered sharp wedges contacting the chip in lines. Real blades will have a finite width in the contact area. FEM analysis shows that even $30 \mu \mathrm{m}$ wide contact areas causes a change in $\sigma_{x x}$ less than $0.6 \%$ and insignificant changes to $\sigma_{y y}$ and $\sigma_{x y}$. Considering the quality of the milling machine used in the fabrication of the setup, these effects are insignificant.

\section{Horizontal blade misalignment: FEM analysis}

FEM is used to describe the influence of a possible misalignment of the blades. The simulations are done with a total misalignment of $\phi= \pm 2^{\circ}$ on each blade in order to

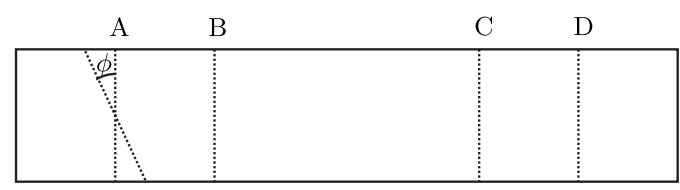

FIG. 11. Top-view schematic of chip and blades (A)-(D). In the FEM analysis, each blade is rotated an angle $\phi$. The stress distribution for different configurations of blade rotation is listed in Table II.
TABLE II. Results of a FEM analysis of blade rotation. The blades are rotated with a worst case misalignment of $\phi=2^{\circ}$ according to Fig. 11. Column 2: the relative difference in the extracted FEM $\sigma_{x x}$ and the analytically calculated stress $\sigma_{x x, \text { an }}$ in the center of the chip surface. Column 3: $\sigma_{y y} / \sigma_{x x}$ at the chip surface in $(x, y)=(1.5 \mathrm{~mm}, 0)$. Column $4: \sigma_{x y} / \sigma_{x x}$ at the chip surface in $(x, y)=(1.5,1.5 \mathrm{~mm})$.

\begin{tabular}{lccc}
\hline \hline \multicolumn{1}{c}{ Configuration } & $\frac{\Delta \sigma_{x x}}{\sigma_{x x, \mathrm{an}}}$ & $\frac{\sigma_{y y, \max }}{\sigma_{x x}}$ & $\frac{\sigma_{x y, \max }}{\sigma_{x x}}$ \\
\hline A,B,C,D: $\phi=0^{\circ}$ & $0.1 \%$ & $0.8 \%$ & $0.3 \%$ \\
B,C,D: $\phi=0^{\circ} ; \mathrm{A}: \phi=2^{\circ}$ & $0.2 \%$ & $0.8 \%$ & $0.2 \%$ \\
B,C: $\phi=0^{\circ} ; \mathrm{A}, \mathrm{D}: \phi=2^{\circ}$ & $0.1 \%$ & $0.8 \%$ & $0.2 \%$ \\
B,C: $\phi=0^{\circ} ; \mathrm{A}: \phi=2^{\circ} ; \mathrm{D}: \phi=-2^{\circ}$ & $0.2 \%$ & $0.8 \%$ & $0.2 \%$ \\
A,C,D: $\phi=0^{\circ} ; \mathrm{B}: \phi=2^{\circ}$ & $0.2 \%$ & $0.8 \%$ & $1.9 \%$ \\
A,D: $\phi=0^{\circ} ; \mathrm{B}, \mathrm{C}: \phi=2^{\circ}$ & $0.4 \%$ & $0.7 \%$ & $3.5 \%$ \\
A,D: $\phi=0^{\circ} ; \mathrm{B}: \phi=2^{\circ} ; \mathrm{C}: \phi=-2^{\circ}$ & $0.2 \%$ & $0.7 \%$ & $0.3 \%$ \\
\hline \hline
\end{tabular}

analyze the effect (see illustration in Fig. 11). The investigation is summarized in Table II, where the ratio of the stress components are listed for different blade configurations. The ratios listed are the maximum values obtained in the resistor area. To obtain the maximum value of $\sigma_{y y} / \sigma_{x x}$, the $(x, y)$ coordinate is $(1.5 \mathrm{~mm}, 0)$. The maximum $\sigma_{x y} / \sigma_{x x}$ value is found in the coordinate $(1.5,1.5 \mathrm{~mm})$.

Notice, that the $\sigma_{x x}$ stress in all cases varies less than $0.5 \%$ with respect to the analytical expression in Eq. (1). It is also seen that the transverse stress, $\sigma_{y y}$, does not depend on the blade misalignment. Thus, we assume that this stress is constant and less than $0.8 \%$ of the $\sigma_{x x}$ stress. A misalignment of the outer blades, A and D in Fig. 11 does not have a significant impact on the stress distribution. However, a misalignment of the inner blades does change the shear stress distribution. As listed in Table II, the largest shear stress, $\sigma_{x y} / \sigma_{x x}=3.5 \%$, is obtained with a rotation in the same direction of the two inner blades.

The above values are extracted for a $2^{\circ}$ horizontal rotation of the blades. This rotation is very large compared to the realistic value, but it is used in order to illustrate the influence. The milling machine used to fabricate the 4PB fixture has a very high precision (precision of $1 \mu \mathrm{m}$ ), thus, it is not expected that a horizontal misalignment influences the stress distribution in the chip.

\section{Vertical blade misalignment}

A vertical rotation of the two inner blades as sketched in Fig. 12 results in a pure torsion of the beam. The resulting shear stress in the surface can be described by ${ }^{29}$

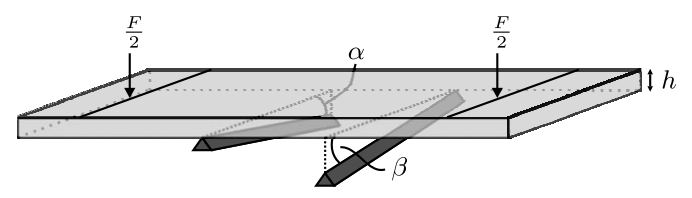

FIG. 12. Schematic of chip exposed to torsion due to vertical misalignment $\alpha$ and $\beta$ of the inner blades. The outer blades each applies a line force of $F / 2$ to the chip of thickness $h$. 


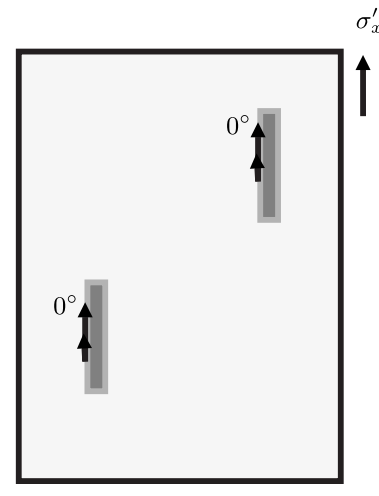

(a)

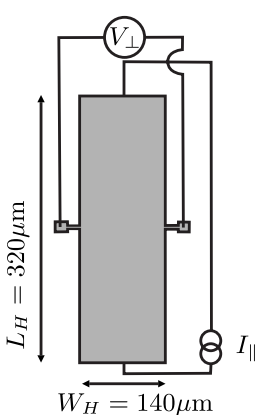

(b)
FIG. 13. (a) Schematic showing the design of the dedicated shear stress sensor chip. The chip consists of two resistors each placed in a corner of the $3 \times 3 \mathrm{~mm}^{2}$ resistor area in the middle of the chip. (b) The current $I_{\|}$is forced through the resistor and a voltage drop $V_{\perp}$ is measured perpendicular to the current direction. The illustrations are not to scale.

$$
\sigma_{x y}=G h \frac{\Delta \psi}{\Delta x},
$$

where the elastic shear modulus $G=Y /(2+2 \nu), \Delta \psi=\alpha+\beta$ in Fig. 12 measured in radians, and the distance between the two inner blades of the chip $\Delta x=12 \mathrm{~mm}$. For example, at a misalignment angle of $\Delta \psi=0.1^{\circ}$, the shear stress component is as large as $\sigma_{x y}=4 \mathrm{MPa}$.

\section{E. Shear stress measurement and discussion}

In order to investigate the actual shear stress component during measurements, we have fabricated a dedicated shear stress sensor chip with the long axis along [100]. The chip design is shown in Fig. 13 where the primed coordinate system is along the $\langle 100\rangle$ crystal axes. A constant current $I_{\|}$is forced through the resistor while the potential drop $V_{\perp}$ perpendicular to the current is measured. The relation between shear stress, $\sigma_{x y}^{\prime}$, and resistance $R_{H}=V_{\perp} / I_{\|}$is

$$
{\frac{\Delta R_{H}}{R_{\square}}}_{[100]}=\frac{1}{2} \pi_{44} \sigma_{x y}^{\prime},
$$

where $R_{\square}$ is the sheet resistance. The result of this measurement is shown in Fig. 14 and proves that there is a nonideal shear stress distribution in the chip. The figure shows a linear relation between the resistance change and the applied stress $\sigma_{x x}^{\prime}$. Thus, when increasing $\sigma_{x x}^{\prime}$, the shear stress also increases. Inserting a measured value of $\pi_{44}=85 \times 10^{-11} \mathrm{~Pa}^{-1}$ (measured at a doping concentration of $N_{A}=2.2 \times 10^{19} \mathrm{~cm}^{-3}$ and $T=30{ }^{\circ} \mathrm{C}$ ) in Eq. (7), we obtain $\sigma_{x y}^{\prime}=3 \mathrm{MPa}$ and $\sigma_{x y}^{\prime}$ $=2 \mathrm{MPa}$ for the two resistors, respectively, at $\sigma_{x x}^{\prime}=85 \mathrm{MPa}$. Thus, we find a shear stress component value which is $3.6 \%$ and $2.5 \%$ of the $\sigma_{x x}^{\prime}$ value. The two resistors are placed in two different corners of the resistor area sketched in Fig. 10, thus, we assume that all resistors experience a shear stress which is in between these two values.

The shear stress is most likely caused by vertical misalignment of the inner blades, since a very small vertical misalignment results in a rather large shear stress component as argued in Eq. (6). In order to accommodate a shear stress of $\sigma_{x y}^{\prime}=3 \mathrm{MPa}$ for $\sigma_{x x}^{\prime}=85 \mathrm{MPa}$, the vertical misalignment of

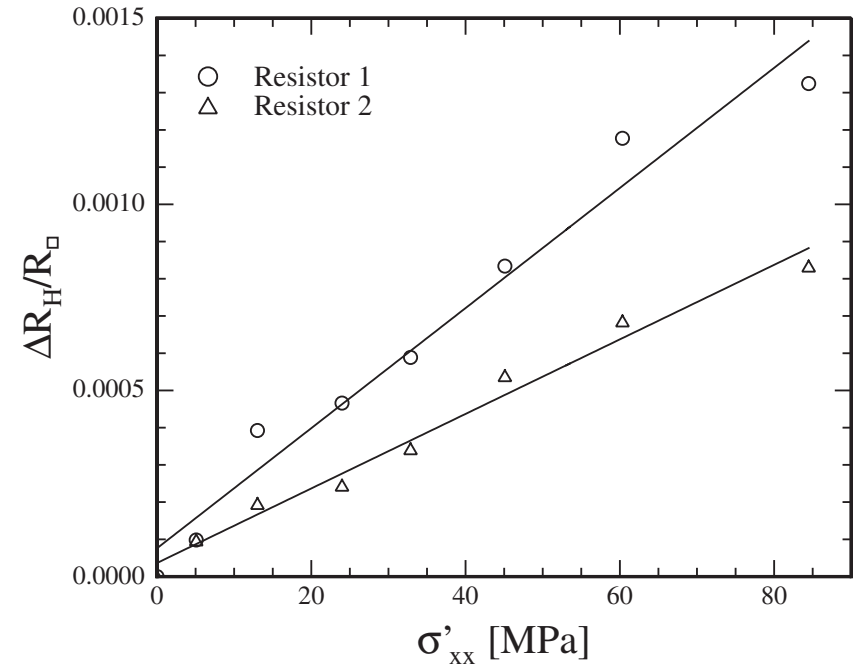

FIG. 14. Plot of the relative resistance change $\Delta R_{H} / R_{\square}$ for the two resistors on the shear stress sensor chip at a temperature of $30^{\circ} \mathrm{C}$ as a function of applied stress $\sigma_{x x}^{\prime}$ along [100]. The resistance change indicates the presence of a shear stress component $\sigma_{x y}^{\prime}$ proportional to $\sigma_{x x}^{\prime}$.

the inner blades is $\Delta \psi=0.07^{\circ}$. A FEM of the shear stress in a chip placed in a $4 \mathrm{~PB}$ fixture as a function of vertical misalignment of the outer blades is performed in Ref. 30. They report a linear relation between the applied force and shear stress until full contact between the vertically misaligned blades and chip is reached. By further increasing the force, the shear stress contribution approaches a constant value. In Fig. 14, it is seen that the shear stress linearly depends on the applied force in the measured stress interval and taking the results from Ref. 30 into account, we find that the inner blades may be misaligned at a larger angle than $\Delta \psi=0.07^{\circ}$. The actual misalignment angle can be measured by increasing the stress even further than what is seen in Fig. 14. Thus, if the shear stress is caused by a vertical misalignment of the inner blades, we conclude that the misalignment is at least $\Delta \psi=0.07^{\circ}$. Another contribution to the shear stress may rise from an in-plane misalignment of the inner blades, as described in Sec. III B. A misalignment of $\phi \approx 2^{\circ}$ of both inner blades need to be present in order to accommodate the measured shear stress and considering the precision of the mechanical equipment used to machine the 4PB (precision $=1 \mu \mathrm{m}$ ), this is not possible. A third contribution is a rotation of the whole PEEK 4PB fixture. The distance between the guiding rails in the bottom of the aluminum housing is $0.15 \mathrm{~mm}$ larger than the width of the 4PB base to accommodate thermal expansion of the two materials. Thus, a rotation of the 4PB fixture is possible. However, due to the very small air gap, the rotation angle is at most $0.3^{\circ}$ and this does not significantly contribute to the shear stress. A fourth contribution to the shear stress is a misalignment of the slider and the base. However, since the two steel pins and guiding holes are specifically fabricated to fit each other with a very small air gap, this is not expected to induce a significant shear stress. Thus, we conclude that the present shear stress in the chip is an effect from a vertical rotation of the inner blades in the $4 \mathrm{~PB}$ fixture. 


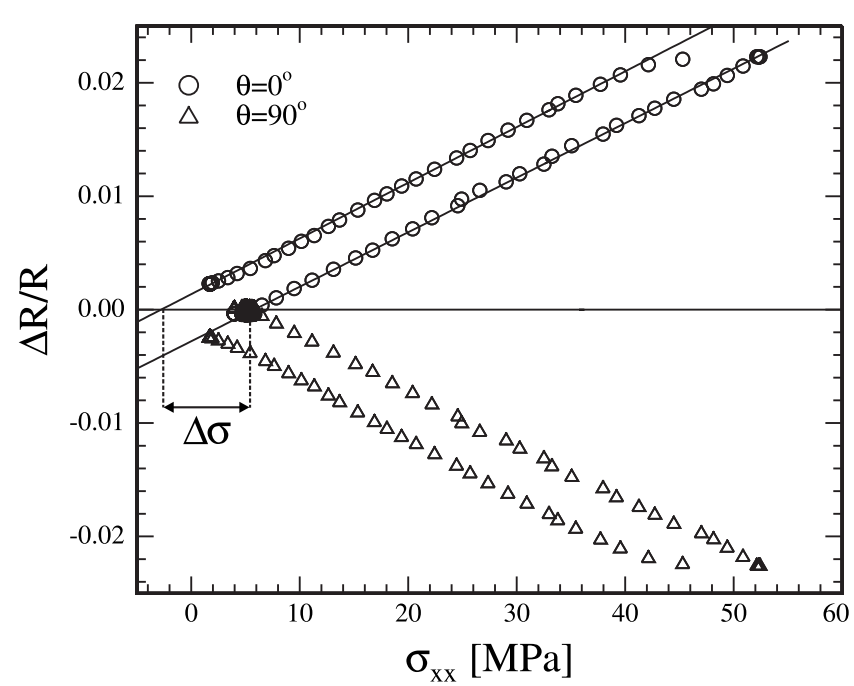

FIG. 15. The relative change in resistance $\Delta R / R$ when increasing and decreasing the load. The measurement data from two resistors, $\theta=0^{\circ}$ and $\theta=90^{\circ}$ in Fig. 7(a), are plotted as a function of the applied stress $\sigma_{x x}$ at $T=30{ }^{\circ} \mathrm{C}$. The solid lines are linear fits to the data. The lateral distance between the fit lines is a measure of the frictional force described by $\Delta \sigma=2 \sigma_{f}$.

\section{F. Electronic setup}

The electronic instruments used to measure the voltage drop and to inject the current are all high precision instruments. The Keithley 2700 multimeter with multiplexer has a resolution of $1 \mu \mathrm{V}$ at a voltage of $1 \mathrm{~V}$ and the Keithley 2400 sourcemeter has a current source accuracy of $0.03 \%$ at $100 \mu \mathrm{A}$. These uncertainties are significantly smaller than the uncertainties described in Sec. III A.

\section{G. Friction}

Frictional forces are expected to be present in the four point bending fixture since it consists of two parts where one is moving (slider) with respect to the other (base). Figure 15 shows a characterization of two resistors during sequential increasing and decreasing loads where the resistance change is measured at each applied load. For both resistors, a hysteresis loop is seen where the measured data are shifted to the right during increasing loads and to the left during decreasing loads. This behavior is explained by the frictional force, $F_{f}$, between the slider and the base, while we conclude from the force diagram sketched in Fig. 16 that the frictional force, $F_{\text {ch }}$, between the $4 \mathrm{~PB}$ base and the aluminum housing does not affect the measurements. The only significant frictional force contribution is from the movement of the slider on the steel pins. The frictional force may be a multivalued

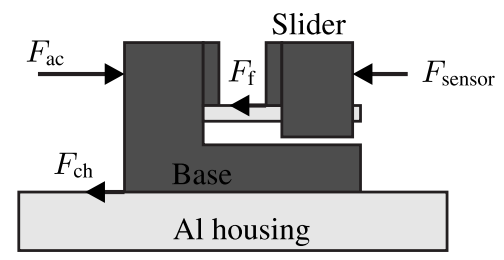

FIG. 16. Force diagram of the $4 \mathrm{~PB}$ fixture. The actuator applies a force $F_{\text {ac }}$ to the $4 \mathrm{~PB}$ fixture and the force on the force sensor is $F_{\text {sensor }}$. The frictional force, $F_{\text {ch }}$, between base and aluminum housing does not contribute to the frictional force seen in Fig. 15. The only contribution to this is the frictional force between base and slider $F_{f}$. function of the velocity $\mathbf{v}$ in a stick-slip fashion. At rest, the magnitude of the applied force must exceed the static stiction-friction force, $F_{f 00}$, to initiate motion, while in motion, the frictional force may be assumed to have a static and a dynamic component, $\mathbf{F}_{f}=\mathbf{F}_{f}(\mathbf{v})=-F_{f 0} \mathbf{v} /|\mathbf{v}|-\beta \mathbf{v}$, where $F_{f 00} \geqslant F_{f 0}$ and $\beta$ is a viscous friction coefficient. In a steady state sequence of measurement steps in a given direction, the stiction-friction force is unimportant, as is the viscous friction, since the important frictional force is found when motion stops. Thus, we expect the relation, $F_{\text {sensor }}=F_{s} \pm F_{f 0}$, between the force, $F_{\text {sensor }}$, measured on the force sensor and the actual force, $F_{s}$, on the sample in the $4 \mathrm{~PB}$ fixture, where the sign depends on the direction of motion.

The force $F_{f 0}$ can be estimated from the width of the hysteresis loop in the stress direction, $\Delta \sigma \simeq 2 \sigma_{f 0} \simeq 8.2 \mathrm{MPa}$, as seen in Fig. 15. This corresponds to a frictional force of $F_{f 0}=0.11 \mathrm{~N}$. If this frictional force is load independent, it does not affect the piezoresistance characterization since only the slopes of the linear fits to the measured resistance change during increasing or decreasing loads are used. We do, however, slightly find different slopes for increasing and decreasing loads, such that they equal the mean slope $\pm 0.6 \%$. This uncertainty must be included in the total uncertainty derived in Sec. III A.

\section{H. Discussion}

In Eq. (3), only the effect of the stress $\sigma_{x x}$ was considered. However, if all in-plane stress components are included, we obtain a relative resistance change $\Delta R / R$ of

$$
\begin{aligned}
\frac{\Delta R}{R}= & \sigma_{x x}\left[\frac{\pi_{11}+\pi_{12}+\pi_{44} \cos (2 \theta)}{2}\right] \\
& +\sigma_{y y}\left[\frac{\pi_{11}+\pi_{12}-\pi_{44} \cos (2 \theta)}{2}\right] \\
& +\sigma_{x y}\left(\pi_{11}-\pi_{12}\right) \sin (2 \theta) .
\end{aligned}
$$

In $p$-type silicon, the piezocoefficient $\pi_{44}$ is much larger than $\pi_{11}$ and $\pi_{12}$, i.e., $\left|\pi_{11} / \pi_{44}\right| \approx 0.8 \%$ and $\left|\pi_{12} / \pi_{44}\right| \approx 4.3 \%$. ${ }^{1}$ Moreover, the maximum value of the shear stress in the chip was measured to be $3.6 \%$ of $\sigma_{x x}$. Thus, it follows from Eq. (8) that the shear stress causes an insignificant error.

However, the contribution from the stress $\sigma_{y y}$ cannot be neglected since its effect is proportional to the large piezocoefficient $\pi_{44}$ in Eq. (8). From the FEM analysis, we conclude that the contribution from $\sigma_{y y}$ is approximately $0.8 \%$ of the contribution from $\sigma_{x x}$. Including this contribution (and the contribution from the uncertainty of the linear fits from Sec. III G) in the uncertainty calculation of the magnitude of $\sigma_{x x}$ in Sec. III A, we conclude that the piezocoefficient $\pi_{44}$ is determined with an uncertainty of $1.8 \%$.

\section{SAMPLE CHARACTERIZATION}

The chips presented in Sec. II E have been characterized in the $4 \mathrm{~PB}$ setup. An example of a measurement series at $T=30{ }^{\circ} \mathrm{C}$ on a chip with a doping concentration of $N_{A}=1.5$ $\times 10^{17} \mathrm{~cm}^{-3}$ is shown in Fig. 17 for increasing loads. The 


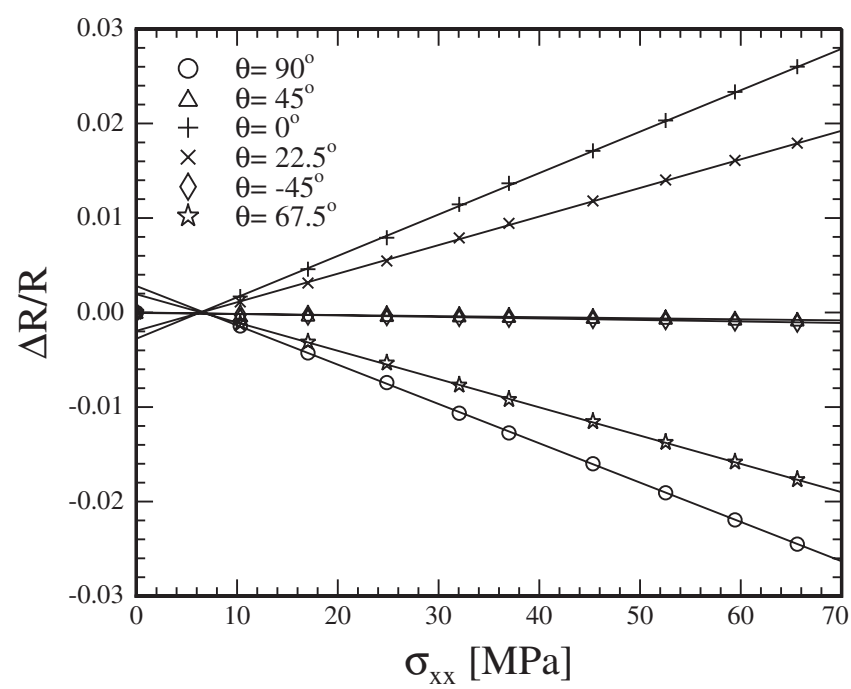

FIG. 17. Relative change in resistance $\Delta R / R$ for the six resistors sketched in Fig. 7(a) as a function of the applied stress. The solid lines are the linear fits to the data series. Notice, the nonzero intercept with the $x$ axis which is due to the frictional force explained in Sec. III G.

figure shows measurements on a chip with six resistors oriented at six different angles, $\theta$, with respect to the stress direction, [110]. The slopes of the linear fits found in Fig. 17 are plotted for each resistor in Fig. 18 as a function of $\cos (2 \theta)$ according to Eq. (3). The slope of the linear fit to these data is proportional to the piezocoefficient $\pi_{44}$, as seen in Eq. (3).

This analysis has been done for samples with the three doping concentrations given in Sec. II E at temperatures from $T=30{ }^{\circ} \mathrm{C}$ to $T=80{ }^{\circ} \mathrm{C}$. The results are shown in Fig. 19 where the measured piezocoefficients are plotted as a function of doping density with the measurement temperature as parameter. The data in the figure are in good agreement with the doping concentration and temperature dependence in previous published results. ${ }^{2}$ The analysis in Sec. III concluded that errors due to shear stress are negligible, the error contribution from $\sigma_{y y}$ is at most $0.8 \%$, and the uncer-

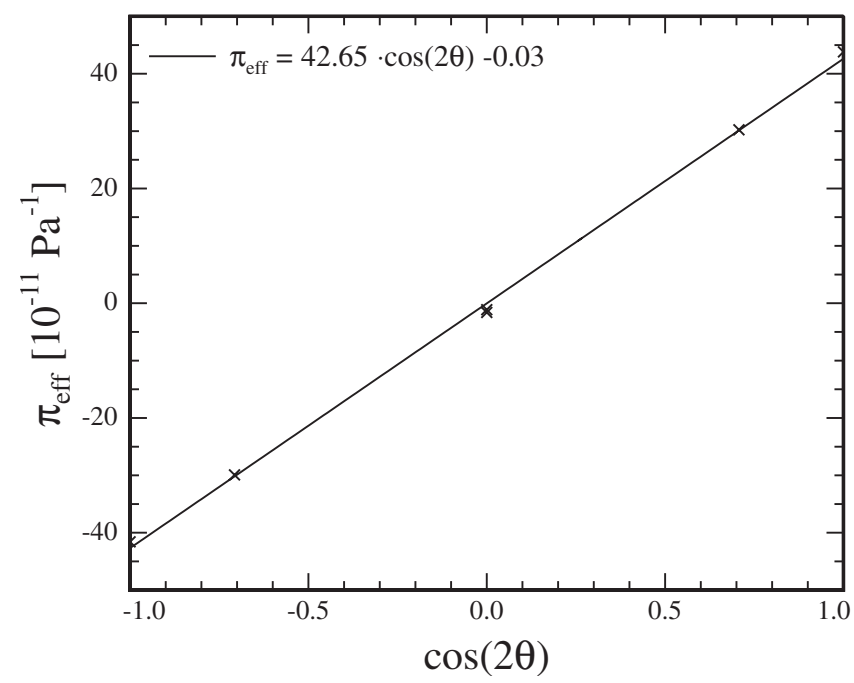

FIG. 18. The slopes of the linear fits in Fig. 17, the effective piezocoefficients $\pi_{\text {eff }}$, are plotted as a function of $\cos (2 \theta)$, where $\theta$ is the angle between the resistor direction and the [110] $\sigma_{x x}$ stress direction. The slope of the linear fit (solid line) is equal to $\pi_{44} / 2$.

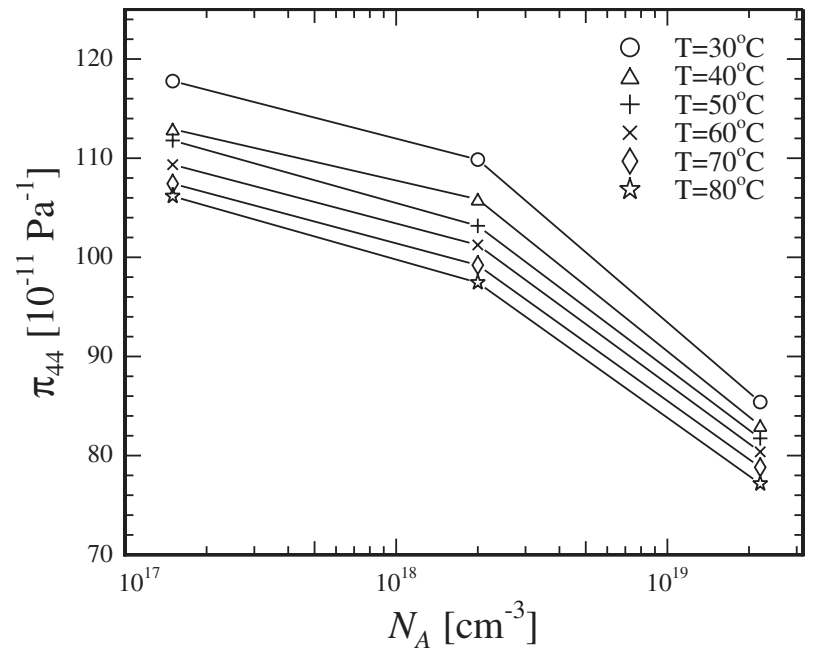

FIG. 19. The measured piezocoefficient $\pi_{44}$ in $p$-type silicon as a function of temperature and doping concentration $N_{A}$.

tainty in the $\sigma_{x x}$ value is $1.5 \%$. The slope of the linear fit to the data is found with an uncertainty of $0.6 \%$, thus, the presented 4PB setup allows measurements of the piezocoefficient $\pi_{44}$ in $p$-type silicon at different doping concentrations and temperatures with an uncertainty of $1.8 \%$.

\section{ACKNOWLEDGMENTS}

Center for Individual Nanoparticle Functionality (CINF) is sponsored by The Danish National Research Foundation. We are grateful for financial support from Grundfos A/S.

${ }^{1}$ C. S. Smith, Phys. Rev. 94, 42 (1954).

${ }^{2}$ O. N. Tufte and E. L. Stelzer, J. Appl. Phys. 34, 313 (1963).

${ }^{3}$ R. C. Jaeger and J. C. Suhling, IEEE Trans. Compon., Hybrids, Manuf. Technol. 16, 925 (1993)

${ }^{4}$ E. Lund and T. Finstad, Proceedings of the 1999 Advances in Electronic Packaging (unpublished), Vol. 12, p. 215.

${ }^{5}$ Grundfos A/S (www.grundfos.com).

${ }^{6}$ Infinion Technologies SensoNor A/S (www.sensonor.no).

${ }^{7}$ P. Yang and R. He, Nat. Nanotechnol. 1, 42 (2006).

${ }^{8}$ J. Richter, O. Hansen, A. Nylandsted Larsen, J. Lundsgaard Hansen, G. F. Eriksen, and E. V. Thomsen, Sens. Actuators, A 123-124, 388 (2005).

${ }^{9}$ Y. Kanda, IEEE Trans. Electron Devices ED-29, 64 (1982).

${ }^{10}$ J. A. Harley and T. W. Kenny, J. Microelectromech. Syst. 9, 226 (2000).

${ }^{11}$ S. I. Kozlovskiy, V. V. Nedostup, and I. I. Boiko, Sens. Actuators, A 133, 72 (2007).

${ }^{12}$ T. Toriyama and S. Sugiyama, J. Microelectromech. Syst. 11, 598 (2002).

${ }^{13}$ C. S. Smith, Macroscopic Symmetry and Properties of Crystals, SolidState Physics Vol. 6 edited by F. Seitz and D. Turnbull (Academic, New York, 1957).

${ }^{14}$ J. Nye, Physical Properties of Crystals, 1st ed. (Oxford University Press, Oxford, 1957).

${ }^{15}$ R. E. Beaty and R. C. Jaeger, IEEE Trans. Compon., Hybrids, Manuf. Technol. 15, 904 (1992).

${ }^{16}$ E. Lund and T. G. Finstad, Rev. Sci. Instrum. 75, 4960 (2004).

${ }^{17}$ J. Bartholomeyczik, S. Brugger, P. Ruther, and O. Paul, IEEE Sens. J. 5, 872 (2005).

${ }^{18}$ V. Weihnacht, W. Bruckner, and C. M. Schneider, Rev. Sci. Instrum. 71, 4479 (2000).

${ }^{19}$ J. Richter, O. Hansen, A. Nylandsted Larsen, J. Lundsgaard Hansen, and E. V. Thomsen, 2006 MRS Fall Meeting, MRS Symposia Proceedings No. 958 (Materials Research Society, Pittsburgh, 2006) p. 133.

${ }^{20}$ C. J. Smithells, Smithells Metals Reference Book, edited by E. A. Brandes and G. B. Brook (Butterworth-Heinemann, Oxford, UK, 1992).

${ }^{21}$ Victrex (www.victrex.com).

${ }^{22}$ Strain Measurement Devices (www.smdsensors.com). 
${ }^{23}$ Newport Corporation (www.newport.com).

${ }^{24}$ Pico Technology (www.picotech.com).

${ }^{25}$ Silvaco Deckbuild (www.silvaco.com).

${ }^{26}$ F. Laermer and A. Schilp, U.S. Patent No. 5,501,893 (August 5, 1994).

${ }^{27}$ Molex Electronics (www.molex.com).
${ }^{28}$ J. J. Wortman and R. A. Evans, J. Appl. Phys. 36, 153 (1965).

${ }^{29}$ S. P. Timoshenko and J. N. Goodier, Theory of Elasticity (McGraw-Hill, New York, 1970).

${ }^{30}$ J. Bartholomeyczik, S. Brugger, S. Kibbel, P. Ruther, and O. Paul, Proceedings of the Eurosensors, 2005 (unpublished), Paper No. TB23. 